\title{
A NOTE ON THE DISTRIBUTIONS OF THE MAXI- MUM OF LINEAR BERNOULLI PROCESSES
}

\author{
SERGEY G. BOBKOV \\ School of Mathematics, University of Minnesota \\ 127 Vincent Hall, 206 Church St. S.E. \\ Minneapolis, MN 55455 USA \\ email: bobkov@math.umn.edu
}

Submitted May 4, 2007, accepted in final form May 5, 2008

AMS 2000 Subject classification: 60J65

Keywords: Linear Bernoulli processes, distribution of the maximum

\section{Abstract}

We give a characterization of the family of all probability measures on the extended line $(-\infty,+\infty]$, which may be obtained as the distribution of the maximum of some linear Bernoulli process.

On a probability space $(\Omega, \mathbf{P})$ consider a linear process

$$
X(t)=a_{0}(t)+\sum_{n=1}^{\infty} a_{n}(t) \xi_{n}, \quad t \in T,
$$

generated by independent, identically distributed random variables $\xi_{n}$ with $\mathbf{E} \xi_{n}=0, \mathbf{E} \xi_{n}^{2}=1$. The coefficients $a_{n}(t)$ are assumed to be arbitrary functions on the parameter set $T$, satisfying $\sum_{n=1}^{\infty} a_{n}(t)^{2}<+\infty$ for any $t \in T$, so that the series (1) is convergent a.s. Define

$$
M=\sup _{t} X(t)
$$

in the usual way as the essential supremum in the space of all random variables with values in the extended real line (identifying random variables that coincide almost surely; cf. Remark 4 below).

We consider the question on the characterization of the family $\mathcal{F}(L)$ of all possible distribution functions $F(x)=\mathbf{P}\{M \leq x\}$ of $M$, assuming that the common law $L$ of $\xi_{n}$ is given. In general, $M$ may take the value $+\infty$ with positive probability, so its distribution is supported on $(-\infty,+\infty]$. Introduce also the collection $\mathcal{F}_{0}(L)$ of all possible distribution functions of $M$ in (2), such that in the series (1), for all $t \in T$,

$$
a_{n}(t)=0, \quad \text { for all sufficiently large } n \text {. }
$$


When $\xi_{n}$ are standard normal, i.e., $L=N(0,1)$, we deal in (1) with an arbitrary Gaussian random process. As is well-known, for the distribution function $F$ of $M, x_{0}=\inf \{x \in \mathbf{R}$ : $F(x)>0\}$ may be finite, and then it is sometimes called a take-off point of the maximum of the Gaussian process. Moreover, $F$ may have an atom at it. But anyway $F$ is absolutely continuous and strictly increasing on $\left(x_{0},+\infty\right)$, which follows from the log-concavity of Gaussian measures (cf. also [C], [HJ-S-D]).

A complete characterization of all possible distributions $F$ in the Gaussian case may be derived from the Brunn-Minkowski-type inequality for the standard Gaussian measure $\gamma_{n}$ on $\mathbf{R}^{n}$ due to A. Ehrhard [E]. It states that, for all convex (and in fact, for all Borell measurable, cf. [Bo2]) sets $A$ and $B$ in $\mathbf{R}^{n}$ of positive measure and for all $\lambda \in(0,1)$,

$$
\Phi^{-1}\left(\gamma_{n}(\lambda A+(1-\lambda) B)\right) \geq \lambda \Phi^{-1}\left(\gamma_{n}(A)\right)+(1-\lambda) \Phi^{-1}\left(\gamma_{n}(B)\right)
$$

where $\Phi^{-1}$ denotes the inverse to the standard normal distribution function on the line. This inequality immediately implies that, if $F$ is non-degenerate, the function $U=\Phi^{-1}(F)$ must be concave on $\mathbf{R}$ in the generalized sense as a function with values in $[-\infty,+\infty)$. But the converse is true, as well.

Indeed, suppose $U=\Phi^{-1}(F)$ is concave on $\mathbf{R}$, and for simplicity let $F$ be non-degenerate and do not assign a positive mass to the point $+\infty$. Then $F$ is strictly increasing on $\left(x_{0},+\infty\right)$, so is its inverse $F^{-1}:\left(F\left(x_{0}\right), 1\right) \rightarrow\left(x_{0},+\infty\right)$. Moreover, the inverse function $U^{-1}=F^{-1}(\Phi)$ is convex and strictly increasing on $\left(U\left(x_{0}\right),+\infty\right)$. Put $M(x)=U^{-1}(x)$ for $x>U\left(x_{0}\right)$, and if $x_{0}$ is finite, $M(x)=x_{0}$ on $\left(-\infty, U\left(x_{0}\right)\right]$. Then $M$ is convex and finite on the whole real line, and therefore admits a representation

$$
M(x)=\sup _{t \in T}\left[a_{0}(t)+a_{1}(t) x\right], \quad x \in \mathbf{R},
$$

for some coefficients $a_{0}(t), a_{1}(t)$. By the construction, $M$ has the distribution function $F$ under the measure $\gamma_{1}$, as was required.

Thus, a given non-degenerate distribution function $F$ belongs to $\mathcal{F}(N(0,1))$, if and only if the function $\Phi^{-1}(F)$ is concave. A similar characterization holds true, when $\xi_{n}$ 's have a shifted onesided exponential distribution with mean zero. Then, $F$ represents the distribution function of $M$ for some coefficients $a_{n}(t)$, if and only if the function $\log F$ is concave. This follows from the log-concavity of the multidimensional exponential distribution (which is a particular case of Prékopa's theorem [P]; cf. also [Bo1] for a general theory of log-concave measures).

In both above examples, for the "if" part it sufficies to consider simple linear processes $X(t)=$ $a_{0}(t)+a_{1}(t) \xi_{1}$. Hence, $\mathcal{F}_{0}(L)=\mathcal{F}(L)$. The situation is completely different, when $\xi_{n}$ have a symmetric Bernoulli distribution $L$, i.e., taking the values \pm 1 with probability $\frac{1}{2}$. This may be seen from:

Theorem 1. Any distribution function $F$, such that $F(x)=0$, for some $x \in \mathbf{R}$, may be obtained as the distribution function of the supremum $M$ of some linear Bernoulli process $X$ in (1) with coefficients, satisfying the property (3).

In turn, the condition (3) ensures that all random variables $X(t)$ in (1) are bounded from below, so is the random variable $M$ in (2). Therefore, the distribution $F$ of $M$ must be onesided. Thus, we have a full description of the family $\mathcal{F}_{0}(L)$ in the Bernoulli case. Removing the condition (3), we obtain a larger family $\mathcal{F}(L)$; however, it is not clear at all how to characterize it. 
One should also mention that in the homogeneous case $a_{0}(t)=0$, much is known about various properties of $M$ in terms of $L$, but the characterization problem is more delicate, and it seems no description or even conjecture are known in all above cases.

For the proof of Theorem 1 one may assume that $\Omega=\{-1,1\}^{\infty}$ is the infinite dimensional discrete cube, equipped with the product Bernoulli measure $\mathbf{P}$. An important property of $\Omega$, which will play the crucial role, is that it represents the collection of all extreme points in the cube $K=[-1,1]^{\infty}$. More precisely, we apply the following statement.

Lemma 2. Any lower semi-continuous function $f:\{-1,1\}^{\infty} \rightarrow(-\infty,+\infty]$ is representable as

$$
f(x)=\sup _{t \in T}\left[a_{0}(t)+\sum_{n=1}^{\infty} a_{n}(t) x_{n}\right], \quad x=\left(x_{1}, x_{2}, \ldots\right),
$$

for some family of the coefficient functions $a_{n}(t)$, defined on a countable set $T$ and satisfying the property (3).

Note any function of the form (4) is lower semi-continuous.

Proof. First, more generally, let $K$ be a non-empty, compact convex set in a locally convex space $E$, and denote by $\Omega$ the collection of all extreme points of $K$. A function $f: \Omega \rightarrow$ $(-\infty,+\infty]$ is representable as

$$
f(x)=\sup _{t} f_{t}(x), \quad x \in \Omega,
$$

for some family $\left(f_{t}\right)_{t \in T}$ of continuous, affine functions on $E$, if and only if

a) $f$ is lower semi-continuous on $\Omega$;

b) $f$ is bounded from below.

This characterization follows from a theorem, usually attributed to Hervé $[\mathrm{H}]$; see E. M. Alfsen [A], Proposition 1.4.1, and historical remarks. Namely, a point $x$ is an extreme point of $K$, if and only if $\bar{g}(x)=g(x)$, for any lower semi-continuous function $g$ on $K$, where $\bar{g}$ denotes the lower envelope of $g$ (i.e., the maximal convex, lower semi-continuous function on $K$, majorized by $g)$.

Clearly, the equality (5) defines a function with properties $a$ ) $-b$ ). For the opposite direction one may use an argument, contained in the proof of Corollary 1.4.2 of [A]. If $f$ is bounded and lower semi-continuous on $\Omega$, put $g(x)=\liminf _{y \rightarrow x} f(y)$ for $x \in \operatorname{clos}(\Omega)$ and $g=\sup _{\Omega} f$ on $K \backslash \operatorname{clos}(\Omega)$. Then $g$ is lower semi-continuous on $K$ and $g=f$ on $\Omega$. By Hervé's theorem, $\bar{g}(x)=g(x)=f(x)$, for all $x \in \Omega$. Since $\bar{g}$ is also convex on $K$, one may apply to it the classical theorem on the existence of the representation

$$
\bar{g}(x)=\sup _{t} f_{t}(x), \quad x \in K,
$$

for some family $\left(f_{t}\right)_{t \in T}$ of continuous, affine functions on $E$ (cf. e.g. [A], Proposition 1.1.2, or $[\mathrm{M}]$, Chapter 11). Thus, restricting this representation to $\Omega$, we arrive at (5). Finally, if $f$ is unbounded from above, write $f=\sup _{n} \min \{f, n\}$ and apply (5) to the sequence $\min \{f, n\}$.

In case of the infinite dimensional discrete cube, the right-hand side of (5) may further be specified. Indeed, any continuous, affine function $g$ on $E=\mathbf{R}^{\infty}$ has the form $g\left(x_{1}, x_{2}, \ldots\right)=$ $a_{0}+\sum_{n=1}^{\infty} a_{n} x_{n}$ with finitely many non-zero coefficients. Therefore, (5) is reduced to the 
relation (4) with some coefficient functions $a_{n}=a_{n}(t)$, that are defined on non-empty, perhaps, uncountable set $T$ and satisfy the property $(3)$.

The latter implies that the sets $T_{N}=\left\{t \in T: a_{n}(t)=0\right.$, for all $\left.n>N\right\}$ are non-empty for all $N \geq N_{0}$ with a sufficiently large $N_{0}$. Define

$$
f_{N}(x)=\sup _{t \in T_{N}}\left[a_{0}(t)+\sum_{n=1}^{\infty} a_{n}(t) x_{n}\right]=\sup _{t \in T_{N}}\left[a_{0}(t)+\sum_{n=1}^{N} a_{n}(t) x_{n}\right],
$$

so that $f=\sup _{N \geq N_{0}} f_{N}$. Since for each point $v=\left(x_{1}, \ldots, x_{N}\right)$ in the finite dimensional discrete cube $\{-1,1\}^{N}$, the second supremum in (6) is asymptotically attained for some sequence of indices in $T_{N}$, one may choose a countable subset $T_{N}(v)$ of $T_{N}$, such that

$$
\sup _{t \in T_{N}}\left[a_{0}(t)+\sum_{n=1}^{N} a_{n}(t) x_{n}\right]=\sup _{t \in T_{N}(v)}\left[a_{0}(t)+\sum_{n=1}^{N} a_{n}(t) x_{n}\right] .
$$

Therefore, the set $T_{N}^{\prime}=\cup_{v \in\{-1,1\}^{N}} T_{N}(v)$ is also countable, is contained in $T_{N}$, and by (6),

$$
f_{N}(x)=\sup _{t \in T_{N}^{\prime}}\left[a_{0}(t)+\sum_{n=1}^{\infty} a_{n}(t) x_{n}\right], \quad \text { for all } x \in\{-1,1\}^{\infty} .
$$

As a result, the supremum in (4) may be restricted to the countable set $\cup_{N} T_{N}^{\prime}$.

Finally, let us note $\Omega$ is compact, so the property $b$ ) is automatically satisfied, when $a$ ) holds. This yields Lemma 2 .

Proof of Theorem 1. According to Lemma 2, we need to show that distributions of lower semi-continuous functions $f$ on $\{-1,1\}^{\infty}$ under the Bernoulli measure $\mathbf{P}$ fill the family of all one-sided distributions on $(-\infty,+\infty]$. In fact, it is enough to consider the functions of the special form $f(x)=\varphi(Q(x))$, where

$$
Q(x)=\sum_{n=1}^{\infty} \frac{x_{n}+1}{2^{n+1}}, \quad x=\left(x_{1}, x_{2}, \ldots\right) \in\{-1,1\}^{\infty},
$$

and where $\varphi:[0,1] \rightarrow(-\infty,+\infty]$ is an arbitrary non-decreasing, left (or, equivalently, lower semi-) continuous function. It is allowed that for some point $p \in[0,1], \varphi$ jumps to the value $+\infty$, and then we require that $\lim _{s \rightarrow p} \varphi(s)=+\infty$, as part of the lower semi-continuity assumption.

The map $Q$ is continuous and pushes forward $\mathbf{P}$ to the normalized Lebesgue measure $\lambda$ on the unit interval $[0,1]$. Hence, $f$ is lower semi-continuous, and its distribution under $\mathbf{P}$ coincides with the distribution of $\varphi$ under $\lambda$.

It remains to see that, for any one-sided probability measure $\mu$ on $(-\infty,+\infty]$, there is an admissible $\varphi$ with the distribution $\mu$ under $\lambda$. Let us recall the standard argument (cf. e.g. [Bi], Theorem 14.1). Introduce the distribution function $F(u)=\mu((-\infty, u]),-\infty<u \leq+\infty$, and define its "inverse"

$$
\varphi(s)=\min \{u: F(u) \geq s\}, \quad 0<s \leq 1 .
$$

Also put $\varphi(0)=\lim _{s \rightarrow 0} \varphi(s)$. Clearly, $\varphi$ is non-decreasing. Given a sequence $s_{n} \uparrow s, 0<s_{n}<$ $s \leq 1$, take minimal values $u_{n}, u$, such that $F\left(u_{n}\right) \geq s_{n}, F(u) \geq s$. We have $u_{n} \uparrow u^{\prime}$, for some 
$u^{\prime} \leq u$. Since $F\left(u^{\prime}\right) \geq s_{n}$, for all $n$, we get $F\left(u^{\prime}\right) \geq s$ and hence $u^{\prime} \geq u$. This shows that $\varphi$ is left continuous. Finally, given $s \in(0,1]$ and $\alpha>\varphi(0)$, by the definition, $\varphi(s) \leq \alpha \Leftrightarrow F(u) \geq s$, for some $u \leq \alpha$. Hence,

$$
\{s \in(0,1]: \varphi(s) \leq \alpha\}=\{s \in(0,1]: F(u) \geq s, \text { for some } u \leq \alpha\}=(0, F(\alpha)] .
$$

Thus, $\varphi$ has the distribution function $F$ under $\lambda$. The proof is now complete.

Remark 3. The statement of Theorem 1 remains to hold in case of arbitrary independent random variables $\xi_{n}$, taking two values, say, $a_{n}$ and $b_{n}$ with probabilities $p_{n}$ and $q_{n}$, satisfying

$$
\prod_{n=1}^{\infty} \max \left\{p_{n}, q_{n}\right\}=0
$$

In this case, the joint distribution $\mathbf{P}$ of $\xi_{n}$ 's represents a product probability measure on $\prod_{n=1}^{\infty}\left\{a_{n}, b_{n}\right\}$ without atoms. Let $a_{n}=-1$ and $b_{n}=1$ (without loss of generality). Then, the map $Q$ in the proof of Theorem 1 pushes $\mathbf{P}$ forward to a non-atomic probability measure $\lambda$ on $[0,1]$, and a similar argument works.

Remark 4. The set $S=S(\Omega, \mathbf{P})$ of all random variables with values in the extended line $(-\infty,+\infty]$ represents a lattice with ordering $X \leq Y$ a.s. Given an arbitrary non-empty collection $\{X(t)\}_{t \in T}$ in $S$, there is a unique element $M$ in $S$, called the essential (or structural) supremum of the family $\{X(t)\}_{t \in T}$, with the properties that

a) $X(t) \leq M$ (a.s), for all $t \in T$;

b) If for all $t \in T$ we have $X(t) \leq M^{\prime}$ (a.s.), $M^{\prime} \in S$, then $M \leq M^{\prime}$ (a.s.)

It is a well-known general fact that $M$ can be represented as a pointwise supremum $M=$ $\sup _{n} X\left(t_{n}\right)$ a.s., for some sequence $t_{n}$ in $T$ (cf. e.g. [K-A]). In particular, the supremum in (2) may always be taken over all $t$ 's from a countable subset of $T$.

Acknowledgement. I am grateful to a referee for valuable comments and references.

\section{References}

[A] E. M. Alfsen (1971), Compact convex sets and boundary integrals. Springer-Verlag, New York, Heidelberg, Berlin.

[Bi] P. Billingsley (1979), Probability and measure. Wiley Series in Probability and Mathematical Statistics. John Wiley \& Sons, New York-Chichester-Brisbane, xiv +515 pp.

[Bo1] C. Borell (1974), Convex measures on locally convex spaces. Ark. Mat., 12, 239-252.

[Bo2] C. Borell (2003), The Ehrhard inequality. C. R. Math. Acad. Sci. Paris, 337, No.10, 663-666.

[C] B. S. Cirel'son, Density of the distribution of the maximum of a Gaussian process. Teor. Verojatnost. i Primenen., 20 (1975), No. 4, 865-873 (Russian).

[E] A. Ehrhard (1983), Symetrisation dans l'espace de Gauss. Math. Scand., 53, No. 2, 281301. 
[H] M. Hervé (1961), Sur les représentations intégrales á l'aide des points extrémaux dans un ensemble compact convexe métrisable. (French) C. R. Acad. Sci. Paris, 253, 366-368.

[HJ-S-D] J. Hoffmann-Jorgensen, L. A. Shepp, and R. M. Dudley (1979), On the lower tail of Gaussian seminorms. Ann. Probab., 7, No. 2, 319-342.

[K-A] L. V. Kantorovich, and G. P. Akilov (1982), Functional analysis. Translated from the Russian by Howard L. Silcock. Second edition. Pergamon Press, Oxford-Elmsford, N.Y., xiv+589 pp.

[M] P. A. Meyer (1966), Probability and potentials. Blaisdell Publ. Co., Toronto.

[P] A. Prékopa (1971), Logarithmic concave measures with application to stochastic programming. Acta Sci. Math. (Szeged), 32, 301-316. 